\title{
Article
}

\section{Mental Health staff views on improving burnout and mental toughness}

Posner, Zoe, Janssen, Jessica and Roddam, Hazel

Available at http://clok.uclan.ac.uk/18559/

Posner, Zoe, Janssen, Jessica ORCID: 0000-0002-5961-2736 and Roddam, Hazel ORCID: 0000-0002-0637-1801 (2017) Mental Health staff views on improving burnout and mental toughness. Journal of Mental Health Training, Education and Practice, 12 (4). pp. 249-259. ISSN 1755-6228

It is advisable to refer to the publisher's version if you intend to cite from the work. http://dx.doi.org/10.1108/JMHTEP-03-2017-0021

For more information about UCLan's research in this area go to http://www.uclan.ac.uk/researchgroups/ and search for < name of research Group>.

For information about Research generally at UCLan please go to http://www.uclan.ac.uk/research/

All outputs in CLoK are protected by Intellectual Property Rights law, including Copyright law. Copyright, IPR and Moral Rights for the works on this site are retained by the individual authors and/or other copyright owners. Terms and conditions for use of this material are defined in the policies page.

\section{CLoK}

Central Lancashire online Knowledge www.clok.uclan.ac.uk

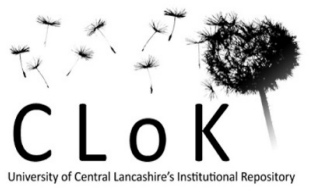


emerald Journal of Mental Health Training, Education and Practice
PUBLISHING

\section{Mental health staff views on improving burnout and mental toughness}

\begin{tabular}{|r|l|}
\hline Journal: & Journal of Mental Health Training, Education and Practice \\
\hline Manuscript ID & JMHTEP-03-2017-0021 \\
\hline Manuscript Type: & Research Paper \\
\hline Keywords: & $\begin{array}{l}\text { Mental health, Intellectual disability, Training, Burnout, Mental Toughness, } \\
\text { Staff Wellbeing }\end{array}$ \\
\hline \multicolumn{2}{|c}{} \\
\hline
\end{tabular}

SCHOLARONE ${ }^{\mathrm{m}}$

Manuscripts 


\title{
Mental health staff views on improving burnout and mental toughness
}

\begin{abstract}
Purpose- Burnout in mental health staff is acknowledged as a major problem. The purpose of this paper is to gain an understanding of mental health staff views on improving burnout and mental toughness in mental health staff.

Design/methodology/approach-Ten participants from two mental health rehabilitation units across the North West of England took part in a Nominal Group Technique (NGT). Participants consisted of mental health workers from varied roles in order to capture views from a multidisciplinary team. The main question posed to the staff was "What strategies and techniques do you think could help improve burnout and mental toughness in mental health staff".
\end{abstract}

Findings- The study revealed that the top three ideas to take forward to help improve burnout and mental toughness in mental health staff were improving the culture/organisation, improving staff wellbeing and education. Additionally, staff were highly motivated and enthusiastic about engaging in discussion about what could be done to improve their wellbeing and the importance of taking this forward.

Originality/Value- This study is unique in involving mental health staff in discussing their ways of improving their mental health. It is also unique as it has found the nine strategies to do this and these could be used in targeted training for mental health staff.

Keywords Mental health, Intellectual disability, Training, Burnout, Mental toughness, Staff wellbeing.

Paper type Research Paper

Introduction

Mental health services have recently seen an increase in demand for their services (Lasalvia et al., 2009). High-volume work demands and targets in these services make it a pressurised environment to work in (Dowthwaite, 2016). Working in mental health is complex, there are pathways for different stages of the individual's condition and there is a wide range of mental health conditions and different roles within the mental health services (National Health service, 2016).

Mental health staff face challenges working in these settings, partly due to the complex nature of the conditions they are working with, including at times 
challenging behaviour from service-users, which can be stressful for the staff (Jenkins et al., 2004). In addition, they also face organisational pressures such as low staffing levels, long shifts and more demands, with low levels of support (Lasalvia et al., 2009). A combination of these factors can be linked to mental health staff experiencing burnout (Lasalvia et al., 2009).

\section{Burnout in mental health staff}

Burnout in mental health staff is acknowledged as a major problem, with as much as $67 \%$ of mental health workers experiencing high levels of burnout (Morse et al., 2012). Staff working in mental health and intellectual disability services are particularly vulnerable to burnout due to factors such as low staffing levels, client pressures and lack of support (Jenkins et al., 2004). The definition of burnout has been described in different ways (Chemise, 1980; Burke et al., 1993). However, the most favoured and accepted definition is one that has multiple dimensions of emotional exhaustion, depersonalisation and reduced personal accomplishment (Maslach, 1996). A reduced sense of personal accomplishment, refers to a negative self-evaluation of work and how well an individual feels they are fulfilling their job role. Emotional exhaustion refers to feeling depleted and fatigued. Depersonalisation includes negative attitudes towards work and clients. Burnout has shown to have a negative impact on the wellbeing of staff (Toppinen-Tanner et al., 2005). Burnout can also undermine the quality of services provided between staff and service-users (Carney et al., 1993). It may also lead to staff being less able to be empathetic, collaborative and attentive to service-users (Corrigan, 1990). Staff with burnout have been linked to having negative attitudes towards service-users, in terms of being distant and rejecting towards them, which in turn has been linked to poorer outcomes in service-users with severe mental health (Holmqvist and Jeanneau, 2006). Therefore, ways of improving levels of burnout in mental health staff is vitally important.

\section{Mental Toughness}

Mental Toughness describes the capacity of an individual to deal effectively with stressors, pressures and challenges, and perform to the best of their ability, irrespective of the circumstances in which they find themselves (Clough et al., 2002). It is suggested that those who can use energy positively during times of crises and pressure and during challenging and demanding situations, are able to have positive attitudes and this reflects high mental toughness (Loehr,1982). Strycharczyk and Clough (2015) have found that mental toughness can be taught and enhanced to individuals from a wide variety of backgrounds. They created the $4 \mathrm{Cs}$ model, which suggests that overall mental toughness is a product of four key factors. This includes seeing challenge as an opportunity, having high levels of self-belief and confidence, being committed to and being able to stick to tasks and believing that you control your own destiny. They have found that mental toughness can be developed through the learning of mental toughness training and techniques (Strycharczyk and Clough, 2015). Mental toughness coaching strategies may include positive thinking, visualisation, goal-setting, attentional control, relaxation and anxiety control (Strycharczyk and Clough, 2015). Crust and Clough (2011) suggests that mental toughness can be enhanced in individuals by providing a challenging yet supportive 
environment, providing an effective social support mechanism and encouraging individual reflection. Therefore, if an individual can deal with pressure and stress more effectively due to being mentally tough, this may improve the levels of burnout in that individual (Gerber et al., 2015). However, this has never been investigated in mental health staff. Previous interventions with mental health have included clinical supervision (Hyrkas, 2005; Edwards et al., 2006), psychosocial interventions (Ewers et al., 2002; Doyle et al., 2007; Redhead et al., 2011) and social support (Jenkins et al., 2004). However, although these interventions showed improvements in some of the dimensions of burnout, these interventions were not informed by staff opinions. It is more beneficial to allow staff to actively be in involved in developing training to improve staff wellbeing and training should be informed by staff opinions and consultation (Coates and Howe, 2015).

It is important that mental health staff wellbeing is taken seriously and that new interventions are developed to help staff to keep themselves well. Interventions that improve an individual's perceived burnout and mental toughness could be hugely beneficial for staff wellbeing as well as having an impact on the care and interactions provided to service-users.

This study was part of a $\mathrm{PhD}$ project with the aim to develop a targeted mental health training package for mental health staff. The staff involved in the study were informed that their ideas would be taken forward to help develop this, which they would be invited too if they were interested. It was explained that the training aimed to improve staff wellbeing by improving self-reported burnout and mental toughness in mental health staff.

\section{Aim of the present study}

This study aimed to gain an understanding of mental health staff views on improving burnout and mental toughness in mental health staff.

\section{Method}

\section{Recruitment}

Participants were identified from two mental health rehabilitation services in the North West of England. Each service had approximately 20 members of staff. The services supported service-users with psychosis as well as other conditions such as depression, anxiety and intellectual disabilities. In the first instance, the manager of the services agreed for a poster advertising the study to be put up. All staff from these services were then sent a letter of invitation, information sheet and consent form in one envelope in their pigeon hole. The manager had requested this as the best way to recruit across these services, due to varying shift patterns and to avoid feeling coerced. The staff then contacted the researcher to express their interest in participating. Ethical approval for this study was granted from the University of Central Lancashire Ethics Committee on 26/09/16. No ethical concerns were identified. 
Participants

Participants were sampled via maximum variation purposive sampling to capture a wide range of perspectives relating to burnout and mental toughness. The sample allowed greater insights to be gained by looking at mental toughness and burnout from different angles, from mental health workers of different age, gender, profession and time working in the service. Inclusion criteria included any individual that had been working in any role in the mental health sector for at least 3 months.

\section{Conduct of the NGT}

The group discussion lasted approximately 2 hours in total. The session included a clear introduction, why the NGT was being used, as well as that a new wellbeing training package for mental health staff is proposed. The researcher gave definitions for burnout (Maslach et al., 1996) and for mental toughness (Clough et al., 2002) in order to clarify that all participants agreed with the definitions. The NGT question was then presented to the group:

"What strategies and techniques do you think could help improve burnout and mental toughness in mental health staff?"

The process consisted of five stages. Stage 1: Participants were asked to write ideas in brief phrases and to work silently and independently. Stage 2: Each individual engaged in a round-robin feedback session to concisely record each idea (without debate from the group). The researcher recorded all ideas from the group on a flip chart that the whole group could see. Stage 3: Each recorded answer was discussed for clarity and importance. Ideas that were similar were discussed and grouped together under one heading, which the staff called themes. Stage 4: Individuals voted privately to prioritise the ideas and ranked these themes from their most favoured action to least favoured action. Stage 5: Individuals reported their priority list back to the group. Votes were tallied and calculated to identify the themes that were rated highest by the group as a whole. The ideas that were most highly rated by the group were most favoured group actions or ideas in response to the question posed at the start.

\section{Results}

A total of 10 participants took part in the Nominal Group Technique (NGT), this included a mix of staff from the two services that were initially approached.

Participants included staff from varying roles and included one clinical lead, two art psychotherapists, one project worker, one occupational therapist/therapy lead, two support workers, two mental health nurses and one assistant psychologist. Of the 10 participants, $7(70 \%)$ were female and $3(30 \%)$ were male. The median age of the female staff was 35 years (range 23-57 years). The median age of the male staff 
was 51 years (range 50-53 years). The median length of time of the participants working in their present post was 2 years 1 month (range 3 months- 24 years) indicating a wide range of experience from different mental health workers in the group.

\section{Definitions of burnout and mental toughness}

The participants were asked if they were happy with the definitions of burnout and mental toughness that were given or wished to offer alternative definitions. The staff not only agreed with these definitions, they also displayed motivation and enthusiasm to discuss these topic areas as the definitions appeared to immediately resonate with the staff.

These definitions developed some interesting discussion. One staff member noted that they

"would like to learn strategies to become mentally tough, so they could use these skills when they needed them, such as in times of stress and pressure". (P6)

Another staff member from another profession also added

"from the definition given I can see areas of mental toughness I would like to improve on straight away' (P3).

It was interesting to note that during the discussion of these definitions, staff thought that there may be a link between mental toughness and burnout and one individual commented that

"improving one of these areas might improve the other". (P2)

\section{The NGT}

The staff had numerous ideas (41 items were recorded) on what strategies and techniques they thought could help improve burnout and mental toughness in mental health staff (Table 1). Ideas that were similar were discussed and grouped together under one heading. The group chose 9 headings for the ideas to be grouped together under; the staff called these headings themes. These consisted of culture/organisation, staff wellbeing, education, teambuilding, positive thinking/feedback, support, relaxation, communication and the environment. Table 1 shows how each specific idea was placed under each theme, the order of themes voted and the definition for the themes.

The most favoured action identified in the NGT was improvements in the culture and organisation. The least favoured action to take forward was the environment. Although the group came up with 9 themes, the importance of each of the 41 individual ideas was acknowledged.

Many important comments were made relating to the NGT question. In particular, throughout the discussion the staff commented on their perception that very little is done to look after or improve staff wellbeing in mental health services. 
One staff member commented on why they thought the existing support in place isn't always useful

"Is it a cultural thing? Supervision is in place but sometimes I don't get chance, we don't complain, just dig in and crack on, when really we should go and get supervision. I've felt quite stressed at times if I have not been able to get supervision because my supervisor off sick or something, I don't go and complain, I just crack on, but really is that the right thing to do?". (P10)

Much of the discussion focussed on why they thought the ideas they had come up with would be beneficial. Some of the quotes from the group discussing the benefits can be seen in Figure 1.

When the votes were tallied and calculated from the group, most actions received similar scores apart from the theme of environment. Therefore, although culture/organisation was the most favoured action, the other themes of staff wellbeing, education, team building, positive feedback, support, relaxation and communication were rated fairly equally by the staff as important actions to take forward. 
Table 1 individual ideas from the group, the themes, order voted and definitions.

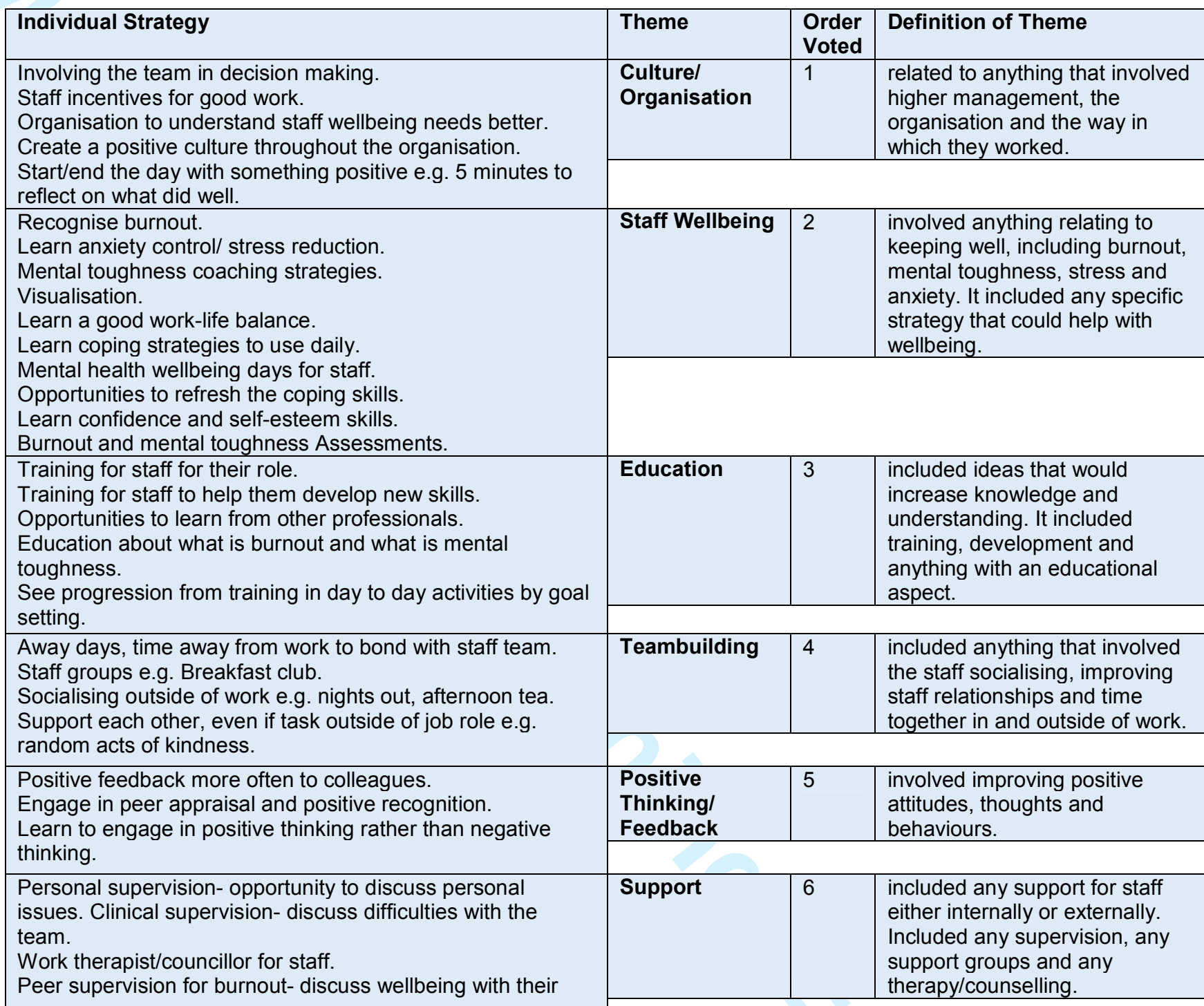

peers.

Set manageable and clear goals throughout the team, and support each other to achieve these.

Use relaxation techniques at work e.g. breathing techniques. Exercise e.g. yoga, tai-chi, walking groups available to staff. Time and space at work to relax e.g. mindfulness sessions. Massage.

Clearly understand roles by open and clear communication. Handovers and de-briefing.

Environment set up so that everyone can do their job effectively e.g. enough computers, chairs, stationary. Provide a quiet space for staff to go to during work. Fresh fruit and water always available to staff to improve nutrition, hydration and energy levels.
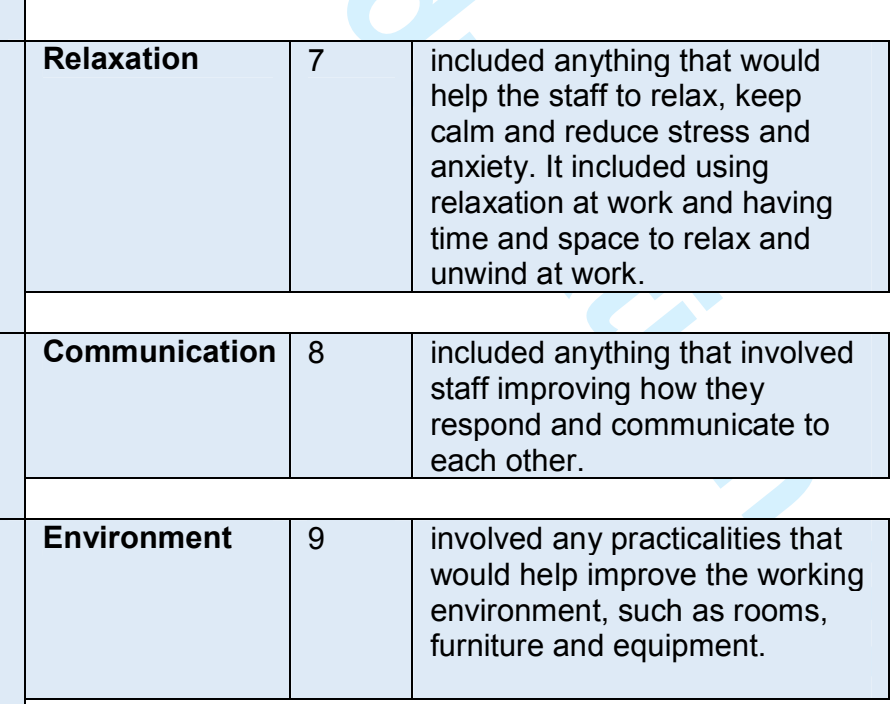
Figure 1 The themes as chosen by the staff, the order the group ranked these, along with quotes from the group.

1. Culture/organisation (most favoured action as voted by the group)

9. Environment (least favoured action as voted by the group)

"Having fresh fruit and water available would improve nutrition, hydration and energy levels and create a nicer environment". (P3)

8. Communication

"sessions like this one would be useful for team decision making as everyone gets to share their ideas and we then come up with an action plan". (P1)

\section{Relaxation}

"it would be great to have the opportunity to engage in relaxation sessions at work". (P5) "feeling valued by the

organisation is important". (P1)

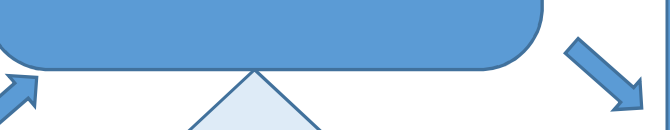
chance to talk about our own wellbeing and burnout". (P2) "we don't' really have the

\section{Staff Wellbeing}

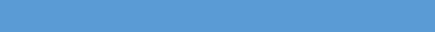

(2) 30 


\section{Discussion}

\section{Improving burnout and mental toughness}

This study shows that staff working in a mental health setting are motivated and enthusiastic to discuss issues around improving their mental health. The top three important themes were: 1) culture/organisation 2) staff wellbeing 3) education. These findings were in line with previously published theoretical and empirical papers. The results have also added value and new knowledge, by generating additional insights to the participants' perspectives and priorities, as well as giving valuable practical pointers for implementation.

The top priority action to take forward from the NGT was improvements in the culture and organisation. This included ideas such as involving the team in decision-making. Previous research identified that it is paramount that new training and initiatives, to improve staff wellbeing and burnout should be informed by mental health staff views and ideas (Coates and Howe, 2015). Therefore, carrying out the NGT was an important process of the development of this training. It is also essential that the staff will be able to see clearly that the training has been created based on their feedback and feel listened too (Coates and Howe, 2015). The staff wanted the organisation to understand staff wellbeing needs and have a better awareness of this issue e.g. training for higher management on staff wellbeing. Previous authors suggested that it is beneficial for management to participate in wellbeing training. Scarnera et al. (2009) found that teaching managers leadership skills and involving them in wellbeing training such as positive thinking workshops is beneficial, as perceived support from managers can affect burnout levels. Therefore, the NGT findings reinforce earlier published work that all staff (including managers) should be involved in wellbeing training.

The second priority action related to staff wellbeing techniques. This included helping staff to recognise and prevent burnout by teaching specific techniques e.g. anxiety control, stress reduction. In previous training 84 participants were encouraged to learn relapse prevention for burnout, which showed improved burnout rates (Salyers et al.,2011). Therefore, the NGT findings concur with other studies that staff wellbeing training should allow staff to learn about burnout and ways of preventing this. Under this theme, staff reported that they would like training on specific techniques that may make them more mentally tough. Mental toughness coaching strategies may be able to improve areas such as performance, resilience and wellbeing (Strycharczyk and Clough, 2015). The development of mental toughness may be achievable through the coaching or training of specific techniques. These techniques include visualisation, positive thinking, attentional control, goal-setting, anxiety control and relaxation (Strycharczyk and Clough, 2015).

The NGT participants emphasised that developing a good work-life balance by learning skills to help with time-management and goal setting would help improve mental toughness and burnout. This supports previous findings that has displayed that goal setting may be useful, as interventions such as clinical supervision, which 
involves group goal setting can be beneficial in reducing burnout in these settings (Edwards et al., 2006).

The mental toughness literature also supports that goal setting and learning to prioritise tasks is beneficial for improving mental toughness. Strycharczyk and Clough (2015) suggests that goal setting helps prioritise and motivate individuals in tasks.

Amongst the staff wellbeing techniques, was confidence and self-esteem training. Positive changes in burnout have been found due to the implementation of selfesteem workshops (Carson et al., 2001). Techniques such as visualisation which can help with confidence and self-esteem are also noted to improve mental toughness (Sheldon et al., 2006). Therefore, self-esteem and confidence training is found to be effective in previous studies and viewed as important in the NGT.

The NGT group also suggested under the heading of staff wellbeing that there should be mental health wellbeing days for staff and regular opportunities to refresh the coping skills they have learned. Practicing wellbeing strategies is important for their effectiveness, with Sheldon and Lyubomirsky (2006) concluding that it may be important to encourage regular practice of such techniques in a training programme for mental health staff. In addition to this, the consideration of refresher or follow up sessions, in order in maintain the effectiveness of the techniques was considered beneficial (Sheldon and Lyubomirsky, 2006).

Staff in the NGT also thought that it would be worthwhile for burnout and mental toughness to be assessed by the workplace, so goals could be made on how to improve. The Mental toughness questionnaire 48 (MTQ48) (Clough et al., 2002) has been found to be useful in assessing which interventions are most useful in improving mental toughness and with different people (Strycharczyk and Clough, 2015). It also allows individuals to see their strengths and limitations and set goals to work on these (Strycharczyk and Clough, 2015). The Maslach burnout Inventory (MBI) (Maslach et al., 1996) also allows staff to see how they score in each of the three dimensions of burnout.

The third priority action was education. During the NGT staff agreed that training and education are essential in improving burnout and mental toughness. The ideas they suggested included increasing knowledge to make them more confident and able to fulfil their responsibilities effectively. Some of the wellbeing training was placed under the heading of staff wellbeing rather than education, as selected by the staff. When mental health staff learn new ways of working with service-users, this can improve burnout, as they feel they have more skills to work effectively with those individuals (Ewers et al., 2002). Others that have explored the use of training to reduce mental health staff burnout have often focussed on teaching staff psychosocial interventions (PSI) as a way of doing so (Ewers et al., 2002; Doyle et al., 2007; Redhead et al., 2011). The skills taught often help staff become more empathetic towards service-users, by increasing their understanding of mental health and learning strategies to work through problems effectively, that service-users may be experiencing (Ewers et al., 2002). The findings are consistent in suggesting that education focussing on new ways of working is valuable. 
Apart from the top three priority themes, the other six themes suggested by the staff were still rated as important and will be incorporated in the proposed training. Theme four- teambuilding- included ideas such as away days and time away from work to bond with the staff team. Group teambuilding has been found to be effective in reducing burnout, as well as improving morale and job satisfaction (RobinsonKurpius and Keim, 1994). Theme five- positive feedback/thinking-suggested that learning to engage in positive thinking rather than negative thinking would be helpful. Training to manage negative emotions, thoughts and increase positive thinking may reduce burnout (Scarnera et al., 2009). Theme six was support. There are positive outcomes in terms of reduced burnout levels in mental health staff with the use of clinical supervision, which provides support to staff (Edwards et al.,2006). However, a recognised problem of using clinical supervision to improve burnout in mental health staff, is that individuals don't always get the opportunity and time out of normal working duties to attend these sessions and they are not always regularly available (White and Roche, 2006). Although relaxation as a technique to improve burnout and mental toughness was prioritised as seventh, the staff were motivated when discussions were had about the benefits of relaxation techniques in the workplace. Goodman and Schorling (2012) support that a mindfulness course can reduce burnout in healthcare providers. As well as training on formal mindfulness practices, the course focussed on how mindfulness could be applied in the workplace, an area that the NGT emphasised as important. Positive and constructive communication was seen as important during the NGT discussion in improving burnout and mental toughness, even though rated eighth in the themes. Communication is important for staff wellbeing, negative communication should be minimised and staff should be encouraged to have discussions that are helpful and constructive (Jenkins et al., 2004). Although voted as the least favoured action to take forward by the group, the staff still selected environment as a theme. Staff require time and space to immerse themselves in the wellbeing techniques they are taught and being away from work in a different environment is beneficial (Razzaque and Wood, 2016).

As discussed the findings are in line with previously published theoretical and empirical papers. However, this study is unique in involving mental health staff in discussing their ways of improving their mental health. It is also unique as it has found nine strategies to do this and these could be used in targeted training for mental health staff.

\section{Implications}

The NGT primarily aimed to find out what strategies and techniques mental health staff thought could be helpful to improve burnout and mental toughness. The findings of this study will be used to develop a staff wellbeing training package for mental health staff. However, in addition to developing ideas for the training the study had other possible implications.

Including staff in the development of training 
An important factor throughout this process is that staff thought it was beneficial for them to be included in the decision making process of what should be included in a wellbeing training package for mental health staff. The importance of making the staff feel their opinions and ideas were valued and that the training would be relevant to them was apparent. According to Coates and Howe (2015) it is important to include mental health staff in developing and designing wellbeing initiatives. The staff reported and agreed that if they were more involved in developing training for themselves, that they would feel more committed and invested in the training. If training is seen as something that is compulsory or 'a means to an end' then staff expressed that they are not fully immersed in the training. Evidence shows that initiatives adapted to the needs and preferences of individual clinical services and staff are more effective (Coffey et al., 2004; McCray et al., 2008). It was clear that from being involved in the development of this wellbeing training package that they were interested and enthusiastic about implementing this and taking it forward.

\section{Using the NGT to start discussions on wellbeing}

The NGT proved to be a beneficial method for aiding the group discussions. It also had additional benefits that were not known prior to the implementation of the NGT. This included the benefits of starting a discussion with the staff about their own wellbeing and what they thought could help improve this. Simply allowing the staff space and time to have the opportunity to discuss wellbeing as a topic was evidently important to the staff that attended. With staff finding that simply talking about the definitions of burnout and mental toughness, sparked enough interest to get them starting to think about their own wellbeing and how to improve this. Discussing wellbeing and a burnout prevention plan with colleagues can be a beneficial tool and is recommended in burnout prevention training (Salyers et al.,2011).

\section{Limitations of the study}

Despite the NGT been a successful method for data collection some limitations of this method are recognised. The NGT can be regimented in the way that it focusses on one question and has a single purpose in a one off meeting. Although during this group the staff engaged in discussion to share ideas and add clarity to points made, it is recognised that compared to other methods the NGT can minimise discussion. Therefore, it is sometimes argued that this can limit the full development of ideas and be less stimulating for the individuals involved (Cantrill et al., 1996). However, as this method allowed for effective data collection with the staff and positive feedback was received from individuals, this method was successful for this study.

\section{Future recommendations}

The findings from the NGT will be of great value in developing a wellbeing training package for staff burnout and mental toughness. It is recommended that the NGT is a beneficial method to capture mental health staff views on what could improve their wellbeing. Further recommendations would be to carry out the NGT with the same question at different services. This could include asking the same question to staff from other mental health rehabilitation services, including those from other organisations. This could include services and organisations from areas outside of 
the North West, across the rest of United Kingdom. It could also include carrying out the NGT with services other than mental health rehabilitation, such as with staff in hospital settings, on acute wards and those working in the community. This would allow for comparison of ideas from different mental health settings and different organisations. It would be interesting to discover if the opinions on strategies and techniques to improve burnout and mental toughness were universal or are specific to services or organisations. This would allow more generalizable wellbeing training packages to be developed. Alternatively, it may suggest that each service has different opinions and that it would be beneficial to always directly include the staff in the development of a wellbeing training package for each individual service.

Conclusion

Staff who engaged in the NGT discussion displayed a clear interest in the topic area of improving staff wellbeing. They offered many ideas and engaged in open discussion on the strategies and techniques that they thought could help improve self-reported burnout and mental toughness in mental health staff. They also valued being engaged in the decision making process and development of the proposed training package. This study showed that mental health staff want to improve the organisational culture towards mental health and their wellbeing and want to be educated about this topic.

\section{References}

Burke R.J and Richardsen A.M. (1993), "Psychological burnout in organizations", in Golembiewski, R.T.(Ed.), Handbook of organizational behavior, Marcel Dekker, New York, NY, pp. 327-363.

Cantrill, J.A., Sibbald, B. and Buetow, S. (1996), "The Delphi and nominal group techniques in health services research", International Journal of Pharmacy Practice, Vol. 4 No. 2, pp. 67-74.

Carney, J., Donovan, R., Yurdin, M. and Starr, R. (1993), "Incidence of burnout among New York City intensive case managers: Summary of findings", Psychosocial Rehabilitation Journal, Vol.16 No.4, pp.25-38.

Carson, J., Kimonis, E., Harman, K. and Kuipers, E. (2001), "What about the workers?", Mental Health Care, Vol. 4 No.10, pp. 344-347.

Chemiss, C. (1980), Staff burnout: Job stress in the human services, Sage, Beverly Hills, CA.

Clough, P. J., Earle, K. and Sewell, D. (2002), "Mental toughness: the concept and its measurement", in Cockerill, I. (Ed.), Solutions in Sport Psychology, Thomson, London, pp. 32-43.

Coates, D.D. and Howe, D. (2015), “The Design and Development of Staff Wellbeing Initiatives: Staff Stressors, Burnout and Emotional Exhaustion at Children and Young 
People's Mental Health in Australia", Administration and Policy in Mental Health, Vol. 42 No. 6, pp.655-663.

Coffey, M., Dugdill, L. and Tattersall, A. (2004), "Stress in social services: mental wellbeing, constraints and job satisfaction", British Journal of Social Work, Vol. 34 No.5, pp. $735-746$.

Corrigan, P.W. (1990), "Consumer satisfaction with institutional and community care", Community Mental Health Journal, Vol.26 No.2, pp.151-165.

Crust, L. and Clough, P.J. (2011), "Developing mental toughness: From research to practice”, Journal of Sport psychology in Action, Vol.2 No 1. pp.21-32.

Dowthwaite, L. (2016), "Happy Hour in IAPT: Improving Workplace Wellbeing", BACP Healthcare Counselling and Psychotherapy Journal, Vol. 16 No. 3, pp. 9-13.

Doyle, M., Kelly, D., Clarke, S. and Braynion, P. (2007), "Burnout: The impact of psychosocial interventions training”, Mental Health Practice, Vol 10 No. 7, pp. 16-19.

Edwards, D., Burnard, P., Hannigan, B., Cooper, L., Adams, J., Juggessur, T., Fothergil, A. and Coyle, D. (2006), "Clinical supervision and burnout: The influence of clinical supervision for community mental health nurses", Journal of Clinical Nursing, Vol. 15 No. 8, pp. 1007-1015.

Ewers, P., Bradshaw, T., McGovern, J. and Ewers, B. (2002), "Does training in psychosocial interventions reduce burnout rates in forensic nurses?" Journal of Advanced Nursing, Vol.37 No.5, pp. 470-476.

Gerber, M., Feldmeth, K., Lang, C., Brand, S., Elliot, C., Holsboer-Trachsler, E. and Puhse, U. (2015), "The relationship between mental toughness, stress and burnout among adolescents: A longitudinal study with Swiss vocational students",

Psychological Reports: Employment Psychology and Marketing, Vol.117 No.3, pp. 703-723.

Goodman, M. J. and Schorling, J. B. (2012), "A mindfulness course decreases burnout and improves well-being among healthcare providers", International Journal of Psychiatry Medicine, Vol. 43 No.2, pp. 119- 128.

Holmqvist, R. and Jeanneau, M. (2006), "Burnout and psychiatric staff's feelings towards patients", Psychiatry Research Journal, Vol. 145 No.2-3, pp.207-213.

Hyrkas, K. (2005), "Clinical supervision, burnout and job satisfaction among mental health and psychiatric nurses in Finland", Issues in Mental Health Nursing, Vol. 26 No. 5, pp. 531-556.

Jenkins, R. and Elliott, P. (2004), "Stressors, burnout and social support: Nurses in acute mental health settings", Journal of Advanced Nursing, Vol. 48 No. 6, pp. $622-$ 631.

Lasalvia, A., Bonetto, C., Bertani, M.E., Bissoli, S., Cristofalo, D., Marrella, G., Ceccato, E., Cremonese, C., De Rossi, M., Lazzarotto, L., Marangon, V., Morandin, I., Zucchetto, M., Tansella, M. and Ruggeri, M. (2009), "Influence of perceived 
organizational factors on job burnout. A survey of staff working in Italian community mental health services", British Journal of Psychiatry, Vol. 195 No. 6, pp. 537-544.

Loehr, J. E. (1982), Athletic excellence: Mental toughness training for sports, Plume, New York, NY.

Maslach, C., Jackson S.E. and Leiter, M.P. (1996), Maslach Burnout Inventory Manual, Consulting Psychologists Press, Palo Alto, CA.

McCray, L. W., Cronholm, P. F., Bogner, H. R., Gallo, J. J. and Neill, R. A. (2008), "Resident physician burnout: is there hope?" Family Medicine, Vol. 40 No.9, pp. 626-632.

Morse, G., Salyers, M.P., Rollins, A.L., Monroe-DeVita, M. and Pfahler, C. (2012), "Burnout in mental health services: A review of the problem and its remediation", Administration and policy mental health, Vol. 39 No. 5, pp. 341-352.

National Health Service. (2016), "A guide to mental health services in England: Glossary of mental health services, teams and care pathways" available at: http://www.nhs.uk/NHSEngland/AboutNHSservices/mental-health-servicesexplained/Pages/services-explained.aspx (accessed 12 December 2016).

Razzaque, R. and Wood, L. (2016), "Exploration of the Effectiveness and Acceptability of a Professional Mindfulness Retreat for Psychiatrists", Mindfulness, Vol.7 No.2, pp. 340-348.

Redhead, K., Bradshaw, T., Braynion, P. and Doyle, M. (2011), "An evaluation of the outcomes of psychosocial intervention training for qualified and unqualified nursing staff working in a low-secure mental health unit", Journal of Psychiatric and Mental Health Nursing, Vol. 18 No. 1, pp. 59-66.

Robinson-Kurpius, S. E. and Keim, J. (1994), "Team building for nurses experiencing burnout and poor morale", Journal for Specialists in Group Work, Vol. 19 No.3, pp. 155-161.

Salyers, M.P., Hudson, C., Morse, G., Rollins, A.L., Monroe-DeVita, M., Wilson, C. and Freeland, L. (2011), "BREATHE: A pilot study of a one-day retreat to reduce burnout among mental health professionals", Psychiatric Services, Vol. 62 No. 2, pp. 214-217.

Scarnera, P., Bosco, A., Soleti, E. and Lancioni, G.E. (2009), "Preventing burnout in mental health workers at interpersonal level: An Italian pilot study", Community Mental Health Journal, Vol. 45 No. 3, pp. 222-227.

Sheldon, K.M. and Lyubomirsky, S. (2006), "How to increase and sustain positive emotion: The effects of expressing gratitude and visualizing best possible selves", Journal of Positive Psychology, Vol. 1 No. 2, pp. 73-82.

Strycharczyk, D. and Clough, P. (2015), Developing mental toughness: coaching strategies to improve performance, resilience and wellbeing, Kogan page Ltd, Great Britain. 
Toppinen-Tanner, S., Ojajärvi, A., Väänänen, A., Kalimo, R. and Jäppinen, P. (2005), "Burnout as a predictor of medically certified sick-leave absences and their diagnosed causes", Behavioral Medicine, Vol.31 No.1, pp.18-27.

White, E. and Roche, M. (2006), "A selective review of mental health nursing in New South Wales, Australia, in relation to clinical supervision", International Journal of Mental Health Nursing, Vol. 15 No. 3, pp. 209-219. 